\title{
Sleep-disordered breathing in patients with myelomeningocele
}

\author{
Daxa M. Patel, MD, ${ }^{1}$ Brandon G. Rocque, MD, MS, ${ }^{1,2}$ Betsy Hopson, MSHA, ${ }^{2}$ \\ Anastasia Arynchyna, MPH, ${ }^{2}$ E. Ralee' Bishop, BS, ${ }^{2}$ David Lozano, MD, ${ }^{3}$ \\ and Jeffrey P. Blount, MD ${ }^{1,2}$
}

\begin{abstract}
1Department of Neurosurgery; ${ }^{2}$ Section of Pediatric Neurosurgery; and ${ }^{3}$ Department of Pulmonology, Children's Hospital of Alabama and University of Alabama at Birmingham, Alabama
\end{abstract}

\begin{abstract}
OBJECT A paucity of literature examines sleep apnea in patients with myelomeningocele, Chiari malformation Type II (CM-II), and related hydrocephalus. Even less is known about the effect of hydrocephalus treatment or CM-II decompression on sleep hygiene. This study is an exploratory analysis of sleep-disordered breathing in patients with myelomeningocele and the effects of neurosurgical treatments, in particular CM-II decompression and hydrocephalus management, on sleep organization.
\end{abstract}

METHODS The authors performed a retrospective review of all patients seen in their multidisciplinary spina bifida clinic (approximately 435 patients with myelomeningocele) to evaluate polysomnographs obtained between March 1999 and July 2013. They analyzed symptoms prompting evaluation, results, and recommended interventions by using descriptive statistics. They also conducted a subset analysis of 9 children who had undergone polysomnography both before and after neurosurgical intervention.

RESULTS Fifty-two patients had polysomnographs available for review. Sleep apnea was diagnosed in $81 \%$ of these patients. The most common presenting symptom was "breathing difficulties" (18 cases [43\%]). Mild sleep apnea was present in 26 cases (50\%), moderate in $10(19 \%)$, and severe in $6(12 \%)$. Among the 42 patients with abnormal sleep architecture, 30 had predominantly obstructive apneas and 12 had predominantly central apneas. The most common pulmonology-recommended intervention was adjustment of peripheral oxygen supplementation (24 cases [57\%]), followed by initiation of peripheral oxygen (10 cases [24\%]).

In a subset analysis of 9 patients who had sleep studies before and after neurosurgical intervention, there was a trend toward a decrease in the mean number of respiratory events (from 34.8 to $15.9, p=0.098$ ), obstructive events (from 14.7 to 13.9, $p=0.85$ ), and central events (from 20.1 to $2.25, p=0.15$ ) and in the apnea-hypopnea index (from 5.05 to $2.03, p$ $=0.038$, not significant when corrected for multiple measures).

CONCLUSIONS A large proportion of patients with myelomeningocele who had undergone polysomnography showed evidence of disordered sleep on an initial study. Furthermore, $31 \%$ of patients had moderate or severe obstructive sleep apnea. Myelomeningocele patients with an abnormal sleep structure who had undergone nonoperative treatment with peripheral oxygen supplementation showed improvement in the apnea-hypopnea index. Results in this study suggested that polysomnography in patients with myelomeningocele may present an opportunity to detect and classify sleep apnea, identify low-risk interventions, and prevent future implications of sleep-disordered breathing.

http://thejns.org/doi/abs/10.3171/2014.11.PEDS14314

KEY WORDS polysomnograph; sleep-disordered breathing; sleep apnea; Chiari malformation Type II; CM-II; myelomeningocele; hydrocephalus

A LTHOUGH the incidence of myelomeningocele has decreased during the last 20 years, it continues to affect approximately 1500 babies annually. ${ }^{14}$ Today, almost $75 \%$ of children born with myelomenin- gocele reach adulthood. ${ }^{3}$ Nevertheless, these individuals continue to face a multitude of medical, physical, and cognitive problems, including a range of sensory and motor impairments, decreased functional mobility, respiratory

ABBREVIATIONS AHI = apnea-hypopnea index; CM-II = Chiari malformation Type II; ECG = electrocardiography; EEG = electroencephalography; EMG = electromyography; $E O G$ = electrooculography; $S D B=$ sleep-disordered breathing.

SUBMITTED June 21, 2014. ACCEPTED November 24, 2014.

INCLUDE WHEN CITING Published online April 3, 2015; DOI: 10.3171/2014.11.PEDS14314.

DISCLOSURE The authors report no conflict of interest concerning the materials or methods used in this study or the findings specified in this paper. 
difficulties, neurological disorders, bowel or bladder dysfunction, orthopedic deformities, intellectual deficits, skin problems, unique allergic reactions, and obesity. ${ }^{16}$ Chiari malformation Type II (CM-II) nearly always accompanies open myelomeningocele, and hydrocephalus is frequently attendant. Many of these abnormalities have the potential to alter respiratory pattern and reflex, leading to sleepdisordered breathing (SDB), ${ }^{8,10,13}$ The patient with myelomeningocele may manifest respiratory abnormalities, such as apnea or hypopnea, that lead to life-threatening conditions.

Existing literature fails to adequately define SDB in the population with myelomeningocele. Even less is known about the effect of a neurosurgical intervention-hydrocephalus treatment or CM-II decompression-on sleep hygiene. The purpose of the present study was to explore the results of polysomnography performed in individuals with myelomeningocele who were monitored at the Children's Hospital of Alabama multidisciplinary spina bifida clinic and to characterize observed cases of SDB. Furthermore, we hoped to better understand the effect of neurosurgical procedures on SDB in patients with myelomeningocele.

\section{Methods}

We performed a retrospective review of all patients seen in the multidisciplinary spina bifida clinic at Children's Hospital of Alabama between March 1999 and July 2013. After institutional board approval was obtained, we reviewed the records of 435 patients with a diagnosis of myelomeningocele and selected those for patients who had undergone polysomnography. Patients were considered eligible for study inclusion if they carried the diagnosis of myelomeningocele and had undergone at least 1 polysomnography evaluation.

Polysomnographs were reviewed for the diagnosis of sleep apnea, type of apnea (central vs obstructive), severity of apnea, total respiratory events, number of obstructive and central events, and apnea-hypopnea index (AHI; Table 1). The AHI is the total number of apnea and hypopnea events divided by the total sleep time, reflecting the severity of sleep apnea. Apnea-hypopnea index values are typically categorized as mild (5-15 events/hour), moderate (16-30 events/hour), or severe ( $>30$ events/hour). We analyzed presenting symptoms and indications for performing sleep studies, as well as pulmonologist recommendations for the management of SDB. Neurosurgeons or other care providers from the multidisciplinary clinic made referrals for a sleep study. There was no specific screening test; instead, patients with concerning signs or symptoms were referred for testing. The diagnosis of CM-II, associated hydrocephalus, and neural tube defect was confirmed via review of MR images. We collected demographic data, including patient age and sex and date of surgical intervention. We also conducted a subset analysis of those who had undergone polysomnography both before and after neurosurgical intervention.

The decision to perform $\mathrm{CM}$ decompression versus shunt placement was based on a patient's presenting symptoms and signs on neurological examination. These therapeutic management options were considered together in our analysis because both have the potential to improve
TABLE 1. Definitions of polysomnographic variables

\begin{tabular}{lc}
\hline \multicolumn{1}{c}{ Event } & \multicolumn{1}{c}{ Definition } \\
\hline Respiratory events & Total number of apnea \& hypopnea events \\
\hline Sleep apnea & $\begin{array}{c}\text { Presence of obstructive, central, or mixed sleep } \\
\text { apnea }\end{array}$ \\
\hline Obstructive apnea & $\begin{array}{c}\text { Event lasting } \geq 10 \text { seconds, characterized by } \\
\text { complete cessation of airflow w/ respiratory } \\
\text { effort persistence }\end{array}$ \\
\hline Central apnea & $\begin{array}{c}\text { Event lasting } \geq 10 \text { seconds, characterized by } \\
\text { complete cessation of airflow w/o respiratory } \\
\text { effort persistence }\end{array}$ \\
\hline Hypopnea & $\begin{array}{c}\text { Event lasting } \geq 10 \text { seconds characterized by } \\
\geq 50 \% \text { reduction in airflow or discernible re- } \\
\text { duction in airflow w/ } 3 \% \text { desaturation, or } \\
\text { termination w/ arousal }\end{array}$ \\
\hline AHI & $\begin{array}{c}\text { Total number of apnea \& hypopnea events divid- } \\
\text { ed by total sleep time; AHI values are typi- } \\
\text { cally categorized as mild (5-15/hr), moderate } \\
\text { (16-30/hr), or severe (>30/hr) }\end{array}$ \\
\hline
\end{tabular}

sleep hygiene given their potential to improve symptoms of hindbrain herniation.

Overnight polysomnography was performed in all 52 patients and consisted of multichannel recordings in the sleep laboratory, such as electroencephalography (EEG), electrooculography (EOG), submental electromyography (EMG), electrocardiography (ECG), oronasal airflow (thermistor- and nasal pressure-based flow measurement), and peripheral pulse oximetry with arterial $\mathrm{O}_{2}$ saturation. Sleep stages were determined using standard EEG, EOG, and EMG criteria. Abnormal respiratory events were determined based on the American Academy of Sleep Medicine criteria..$^{5,711}$

All statistical analysis was performed using SPSS version 21.0 (SPSS Inc.). Descriptive statistics for the study population are expressed as the mean \pm standard deviation and 95\% confidence interval. Preoperative and postoperative polysomnography values were compared using the Wilcoxon test for paired samples. A p value $<0.05$ was deemed statistically significant. Bonferroni correction was applied to correct for multiple measures in this retrospective sample.

\section{Results}

Of the 435 patients with myelomeningocele, 52 had polysomnographs available for review. Patient age at the time of initial study ranged from 2 months to 24.5 years (median 7.4 years, mean $8.3 \pm 0.9$ years). There were 25 males (48\%) and 27 females (52\%; Table 2). Sleep apnea was diagnosed in $42(81 \%)$ of the 52 patients: mild sleep apnea in 26 cases (50\%), moderate in $10(19 \%)$, and severe in $6(12 \%)$. Among the patients with diagnosed apnea, 30 (71\%) had predominantly obstructive apneas and 12 (29\%) had predominantly central apneas.

The most common presenting symptom was breathing difficulties (18 cases [43\%]), including apnea (13 cases [31\%]), blue spells (2 cases [4.8\%]), shortness of breath (1 case [2.4\%]), and other complaints (2 cases [4.8\%]). Other 
TABLE 2. Spina bifida characteristics and sleep findings in $\mathbf{5 2}$ patients with myelomeningoceles

\begin{tabular}{cc}
\hline \multicolumn{1}{c}{ Variable } & No. $(\%)$ \\
\hline Mean age in yrs & $8.3 \pm 0.9$ \\
\hline Sex & $25(48)$ \\
\hline Male & $27(52)$ \\
\hline Female & $42(81)$ \\
\hline Sleep apnea & $26(50)$ \\
\hline Mild apnea & $10(19)$ \\
\hline Moderate apnea & $6(12)$ \\
\hline Severe apnea & $30(71)$ \\
\hline Obstructive apnea & $12(29)$ \\
\hline Central apnea & $18(43)$ \\
\hline Presenting symptoms & $13(31)$ \\
\hline Breathing difficulties & $2(4.8)$ \\
\hline Apnea & $1(2.4)$ \\
\hline Blue spells & $2(4.8)$ \\
\hline Shortness of breath & $6(14)$ \\
\hline Other & $4(9.5)$ \\
\hline Snoring & $2(4.8)$ \\
\hline Excessive daytime sleepiness & $1(2.4)$ \\
\hline Choking &
\end{tabular}

presenting symptoms included snoring (6 cases [14\%]), excessive daytime sleepiness (4 cases [9.5\%]), choking (2 cases [4.8\%]), and irritability (1 case [2.4\%]).

For the 42 patients with diagnosed SDB, we evaluated specific recommendations made by the pulmonologist. The most commonly recommended intervention was peripheral oxygen supplementation: adjustment in 24 cases (57\%) and initiation in 10 cases (24\%; Table 3). Other common recommendations were neurosurgical evaluation in 9 cases (21\%), otolaryngology evaluation in 7 cases (17\%), interval follow-up with another polysomnograph in 4 cases (10\%), and cardiac and pulmonary evaluation in 1 case (2.4\%). The follow-up period for the 52 patients ranged from 3 to 36 months. Of the 52 patients, 37 had repeat polysomnography studies available for review.

Notably, of the 9 patients for whom the pulmonologists recommended neurosurgical evaluation, 4 went on to have a neurosurgical procedure. All 4 of these patients also had postoperative polysomnography, and thus are included in the neurosurgical subgroup discussed below.

We examined the outcomes of the 33 patients who were recommended for nonneurosurgical intervention in further detail. Postintervention sleep studies were available in 24 of these patients (Table 4). Significant changes in the AHI occurred in 17 patients after adjustment and initiation of peripheral oxygen: decrease from $10.6 \pm 3.3$ to 3.3 $\pm 1.3(\mathrm{p}=0.007)$. After otolaryngology evaluation, followup polysomnography studies without interval intervention, and cardiac and pulmonary evaluations, no respiratory sleep variables showed a statistically significant change.

Nine patients (7 males [78\%] and 2 females [22\%]) underwent neurosurgical intervention and had pre- and postoperative polysomnography studies. All 9 patients
TABLE 3. Pulmonologist recommendations for the management of SDB among 42 patients

\begin{tabular}{lc}
\hline \multicolumn{1}{c}{ Therapy } & No. (\%) \\
\hline Adjustment of peripheral $\mathrm{O}_{2}$ & $24(57)$ \\
\hline Initiation of peripheral $\mathrm{O}_{2}$ & $10(24)$ \\
\hline Neurosurgical evaluation & $9(21)$ \\
\hline Otolaryngology & $7(17)$ \\
\hline Continuation of FU w/ another polysomnograph & $4(10)$ \\
\hline Cardiology evaluation & $1(2.4)$ \\
\hline Pulmonary evaluation & $1(2.4)$ \\
\hline
\end{tabular}

$\mathrm{FU}=$ follow-up.

were chosen for the neurosurgical intervention based on symptoms. None of the operative reports for these patients specifically mentioned polysomnography findings as an indication for surgery; however, in 3 cases symptoms such as stridor or desaturation were among the indications for surgery. The surgical intervention was CM-II decompression in 6 patients and ventriculoperitoneal shunt placement for hydrocephalus management in 4 patients ( 1 patient underwent both procedures). Age at surgery among these patients ranged from 16 days to 8.6 years. (median $4.2 \pm 4.14$ years). Both pre- and postsurgery data are compared in Table 5 and Figs. 1 and 2. Sleep apnea had been diagnosed prior to neurosurgical intervention in 7 patients (78\%), while sleep studies in the other 2 patients $(22 \%)$ had not shown sleep apnea. After surgery, there was a trend toward a decrease in the mean number of respiratory events (from $34.8 \pm 27.9$ to $15.9 \pm 14.6, \mathrm{p}=0.098$ ), obstructive events (from $14.7 \pm 13.5$ to $13.9 \pm 14.6, p=0.85$ ), and central events (from $20.1 \pm 24.7$ to $2.3 \pm 4.2, \mathrm{p}=0.15$ ). There was also a decrease in the AHI from $5.0 \pm 3.9(95 \%$ CI $2.0-8.1)$ to $2.0 \pm 1.9$ (95\% CI $0.6-3.5 ; \mathrm{p}=0.038$ ). This decrease did not maintain statistical significance when corrected for multiple measures. Tables 6 and 7 illustrate the change in sleep parameters in patients undergoing CM-II decompression and shunt placement, respectively.

TABLE 4. Respiratory sleep variables pre- and postintervention in 24 patients who underwent nonneurosurgical management

\begin{tabular}{|c|c|c|c|}
\hline \multirow[b]{2}{*}{ Respiratory Variable } & \multicolumn{2}{|c|}{ Mean No. of Events } & \multirow{2}{*}{$\begin{array}{c}p \\
\text { Value }\end{array}$} \\
\hline & Preop & Postop & \\
\hline \multicolumn{4}{|c|}{$\begin{array}{l}\text { Adjustment \& initiation of periph- } \\
\quad \text { eral } \mathrm{O}_{2}(17 \text { patients })\end{array}$} \\
\hline Respiratory events & $63.5 \pm 22.3$ & $20.0 \pm 7.22$ & 0.054 \\
\hline Obstructive events & $18.8 \pm 9.23$ & $10.8 \pm 5.7$ & 0.439 \\
\hline Central events & $44.7 \pm 18.3$ & $9.2 \pm 4.1$ & 0.035 \\
\hline $\mathrm{AHI}$ & $10.6 \pm 3.3$ & $3.3 \pm 1.3$ & $0.007^{*}$ \\
\hline \multicolumn{4}{|c|}{$\begin{array}{l}\text { Interval polysomnograph w/o in- } \\
\text { tervention (7 patients) }\end{array}$} \\
\hline Respiratory events & $28.6 \pm 8.3$ & $74.3 \pm 31.4$ & 0.236 \\
\hline Obstructive events & $20.0 \pm 7.7$ & $36.8 \pm 17.1$ & 0.403 \\
\hline Central events & $8.6 \pm 2.8$ & $37.4 \pm 17.1$ & 0.155 \\
\hline $\mathrm{AHI}$ & $15.1 \pm 11.1$ & $9.0 \pm 2.7$ & 0.575 \\
\hline
\end{tabular}

* Statistically significant. 
TABLE 5. Respiratory sleep variables pre- and postoperatively in 9 patients who underwent neurosurgical intervention

\begin{tabular}{lccc}
\hline & \multicolumn{2}{c}{ Mean No. of Events $(95 \% \mathrm{Cl})$} & \multirow{2}{*}{ p Value } \\
\cline { 2 - 3 } Respiratory Variable & Preop & Postop & 0.098 \\
\hline Respiratory events & $34.8(14.8-54.7)$ & $15.9(5.4-26.4)$ & 0.85 \\
\hline Obstructive events & $14.7(5.0-24.4)$ & $13.9(3.4-24.3)$ & 0.15 \\
\hline Central events & $20.1(3.0-32.8)$ & $2.3(-0.9$ to 5.5$)$ & $0.038^{*}$ \\
\hline AHI & $5.0(2.0-8.1)$ & $2.0(0.6-3.5)$ & \\
\hline
\end{tabular}

* Fails Bonferroni correction for multiple measures.

\section{Discussion}

The purpose of this study was to characterize the sleep hygiene of patients with myelomeningocele through a retrospective review and data analysis. The results provided hypotheses for future studies aimed at providing better care for patients with myelomeningocele.

\section{Sleep-Disordered Breathing in Patients With Myelomeningocele}

Approximately $81 \%$ of patients with myelomeningocele who had undergone polysomnography were classified as having sleep apnea. Since these patients underwent polysomnography given signs or symptoms prompting referral, this rate does not represent the incidence of SDB in our myelomeningocele population, but rather has the potential to be an underestimate. However, the 42 patients with diagnosed sleep apnea represent $9.6 \%$ of the clinic population (435 patients). In the general pediatric population, obstructive sleep apnea is estimated to have a prevalence of approximately $4 \% .{ }^{2}$ Given that the observed prevalence in our study is probably an underestimate-yet is more than

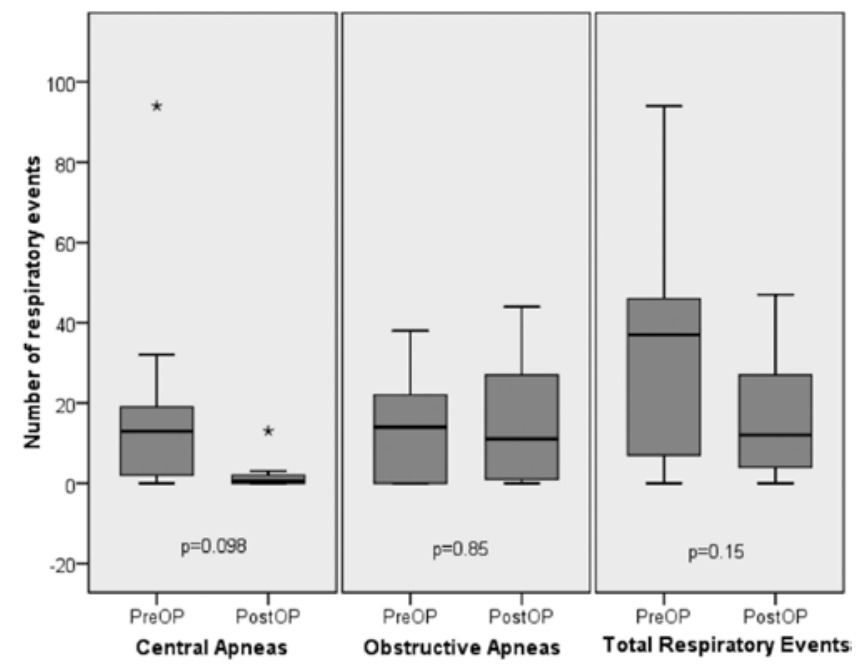

FIG. 1. Boxplot showing preoperative (PreOP) and postoperative (PostOP) findings in patients with myelomeningocele who underwent surgical intervention. Central events, obstructive events, and total respiratory events demonstrated improvement after surgery. The box itself contains the middle $50 \%$ of the data. The upper hinge, or edge, of the box indicates the 75th percentile of the data set, and the lower hinge indicates the 25th percentile. The bold line in the box indicates the median value. Whiskers indicate the minimum and maximum data values, unless outliers are present, in which case the whiskers extend to a maximum of 1.5 times the interquartile range. Points outside the ends of the whiskers are outliers or suspected outliers, which are represented by asterisks. double the rate seen in the general population-we suggest that this condition may be a very common unrecognized source of morbidity in patients with myelomeningocele.

Furthermore, sleep apnea was either moderate or severe in $31 \%$ of our population. The severity of sleep apnea is important because it reflects the number of abnormal events or episodes per hour. A higher number of apneas or hypopneas per hour is associated with more complications and greater morbidity., ${ }^{4,21}$ Our severity data underscore the significance of morbidity associated with SDB in patients with myelomeningocele.

Few studies in the literature address SDB in the myelomeningocele population. Waters et al. performed polysomnography in 83 patients with myelomeningocele, from a population of 109, and found that breathing during sleep was abnormal in 52 cases (62\%), mildly abnormal in 35 (42\%), and moderately and/or severely abnormal in 17 $(20 \%){ }^{22}$ More recently, Alsaadi et al. evaluated 16 children with CM-II and neural tube defect. ${ }^{1}$ These authors do not mention how these patients were selected or how many patients made up the population. Sleep apnea was diagnosed in $11(69 \%)$ of the 16 patients. Data in our study corroborate findings in these earlier studies. There is a

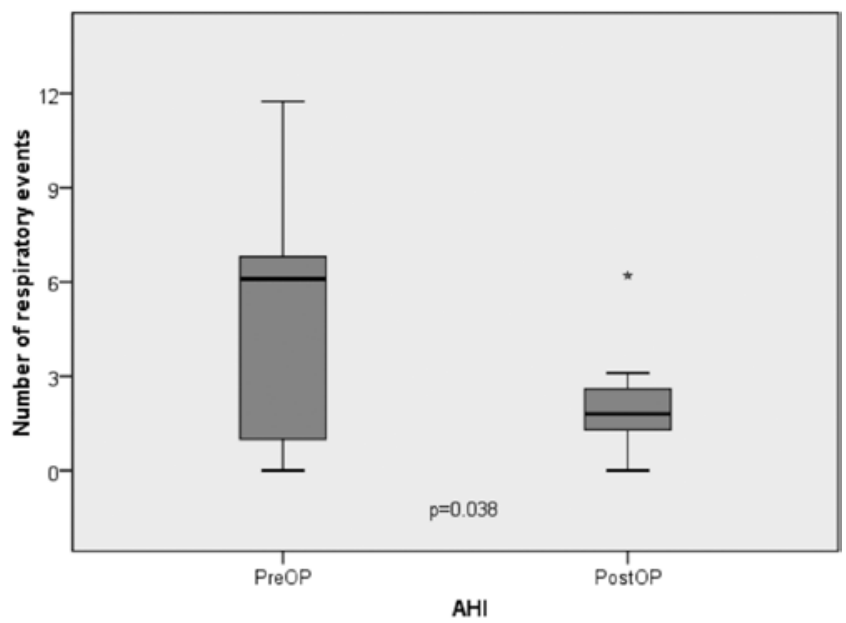

FIG. 2. Boxplot showing preoperative (PreOP) and postoperative (PostOP) findings in patients with myelomeningocele who underwent surgical intervention. Improvement in the $\mathrm{AHI}$ is not statistically significant $(p=0.038)$ after Bonferroni correction for multiple measures. The box contains the middle $50 \%$ of the data. The upper hinge of the box indicates the 75th percentile of the data set, and the lower hinge indicates the 25th percentile. The bold line in the box indicates the median value. Whiskers indicate the minimum and maximum data values, unless outliers are present, in which case the whiskers extend to a maximum of 1.5 times the interquartile range. Points outside the ends of the whiskers are outliers or suspected outliers, which are represented by asterisks. 
TABLE 6. Respiratory sleep variables pre- and postoperatively in 6 patients who underwent $\mathrm{CM}-\mathrm{Il}$ decompression

\begin{tabular}{lccc}
\hline \multirow{2}{*}{ Respiratory Variable } & \multicolumn{2}{c}{ Mean No. of Events } & \\
\cline { 2 - 3 } & Preop & Postop & p Value \\
\hline Respiratory events & $20.3 \pm 19.2$ & $10.8 \pm 10.4$ & 0.409 \\
\hline Obstructive events & $13.3 \pm 13.3$ & $5.8 \pm 6.5$ & 0.752 \\
\hline Central events & $7.0 \pm 8.0$ & $2.5 \pm 5.2$ & 0.096 \\
\hline AHI & $3.6 \pm 3.5$ & $1.2 \pm 1.0$ & 0.340 \\
\hline
\end{tabular}

higher prevalence of SDB among patients with myelomeningocele than in the general pediatric population.

\section{Mechanism of SDB in Patients With Myelomeningocele}

The underlying mechanisms of sleep apnea in patients with myelomeningocele can be attributed to distortion of the brainstem and the structures in proximity. The presence of CM-II, myelomeningocele, and associated hydrocephalus causes compression of the respiratory nuclei in the medulla and impingement of the lower cranial nerves controlling vocal cords and bulbar muscles involved in pharyngeal airway patency. ${ }^{8,10,15}$ Furthermore, children with myelomeningocele have dysfunction of not only the central chemoreceptors, but also the peripheral chemoreceptors causing arousal deficits to respiratory stimuli. 9,18,20,22 These patients appear to have normal breathing during wakefulness but exhibit prolonged expiratory apnea with cyanosis during sleep that manifests mainly as an obstructive problem..$^{10,13,15}$ We have observed that $71 \%$ of patients with SDB have apnea that is predominantly obstructive in nature. This finding suggests that perhaps lower cranial nerve dysfunction plays a larger role than central respiratory center dysfunction.

\section{Symptoms of SDB}

One of the most important initial steps in diagnosing SDB in children is early recognition of symptoms. In our patients, the most frequently reported symptoms were apnea, blue spells, shortness of breath, snoring, excessive daytime sleepiness, choking, and irritability. These are the same symptoms that suggest SDB in the general pediatric population. ${ }^{19}$ These findings in a patient with myelomeningocele may be a useful trigger for evaluation via polysomnography.

\section{Interventions for SDB in Patients With Myelomeningocele}

After polysomnography, the treating pulmonologist's most frequent recommendation was the initiation or adjustment of peripheral oxygen supplementation. Evaluation of the available sleep studies following these interventions suggests that this treatment is effective in improving the AHI. This high level of treatment success with a very low risk suggests that oxygen supplementation may be a very reasonable first-line treatment for myelomeningocele patients with sleep apnea.

In the present study, a subset of 9 patients had sleep studies available for comparison before and after posterior fossa decompression or shunt placement. Sleep studies were not performed specifically to assess response to neurosurgical treatment, nor was surgery performed with the
TABLE 7. Respiratory sleep variables pre- and postoperatively in 4 patients who underwent shunt placement

\begin{tabular}{lccc}
\hline \multirow{2}{*}{ Respiratory Variable } & \multicolumn{2}{c}{ Mean No. of Events } & \\
\cline { 2 - 3 } & Preop & Postop & p Value \\
\hline Respiratory events & $47.8 \pm 38.4$ & $19.5 \pm 21.9$ & 0.885 \\
\hline Obstructive events & $13.0 \pm 17.9$ & $18.8 \pm 20.6$ & 0.06 \\
\hline Central events & $34.8 \pm 41.6$ & $0.8 \pm 1.5$ & 0.652 \\
\hline AHI & $6.0 \pm 4.8$ & $2.8 \pm 2.6$ & 0.641 \\
\hline
\end{tabular}

goal of improving sleep hygiene. Thus, interpretation of these data is limited to the observation of a potential association between the neurosurgical procedure and a change in polysomnography parameters. In fact, we did observe a trend toward improvement in all polysomnography parameters following neurosurgical treatment. However, 2 of the 9 patients had initiation or adjustment of peripheral oxygen supplementation as well as a neurosurgical intervention. Thus, it is possible that the observed trend was attributable to oxygen supplementation and not a direct benefit of surgery. From these data, we cannot conclude that CM-II decompression or ventriculoperitoneal shunting has any effect on sleep hygiene.

\section{Significance of the Findings}

Data in the present study show that SDB is a common condition occurring in children with myelomeningocele. Eighty-one percent of patients with symptoms concerning for abnormal sleep hygiene had polysomnography findings consistent with SDB. Furthermore, the patients with SDB represented $9.6 \%$ of the total clinic population. Both of these rates are higher than those seen in the general pediatric population. Severe cases of SDB can be life-threatening and can lead to death if left undiagnosed and untreated. ${ }^{12}$ A survey of American and Canadian spina bifida clinics documents $11 \%$ of deaths from respiratory failure and only $8 \%$ evaluations for SDB. ${ }^{9}$ The present study and previously published literature suggest that SDB in patients with myelomeningocele is highly prevalent, underrecognized, underreported, and undertreated. Therefore, a rigorous prospective trial screening myelomeningocele patients for SDB could be very informative and beneficial.

In addition, patients with myelomeningocele have been noted to display a stereotypical spectrum of cognitive difficulties. ${ }^{6}$ Adults with SDB also display measurable cognitive decline ${ }^{17}$ Given the severity of brain abnormalities that CM-II represents, one can attribute several potential causes to the myelomeningocele cognitive phenotype. However, one contribution to this phenotype may be unrecognized SDB. If we can identify SDB in patients with myelomeningocele and treat them with low-cost interventions such as oxygen supplementation, we may be able to improve cognition for a subset of these patients.

Present findings reinforce the practice of performing polysomnography in patients with myelomeningocele who present with respiratory symptoms, in particular breathing difficulties, snoring, excessive daytime sleepiness, choking, and irritability. Furthermore, if the polysomnography results were abnormal, a trial of conservative management with the initiation or adjustment of peripheral oxygen 
would be reasonable. If symptoms or polysomnography abnormalities persisted, evaluation by a multidisciplinary team including otolaryngology and neurosurgery personnel would be beneficial.

\section{Study Limitations}

This study has several limitations. It is a retrospective overview of findings in our myelomeningocele population and represents an initial attempt to understand their sleep hygiene. Patients who underwent an initial sleep study were symptomatic and thus cannot provide information on the prevalence of SDB in the entire myelomeningocele population.

There are also several limitations to polysomnography. Recording conditions may not reflect what happens during a regular night in the patient's home. Furthermore, recordings are based on one night's sleep patterns, which may not be enough to capture SDB. Sporadic events can be missed with a single-night polysomnography. External factors that disturb the patient's sleep can be present in the home but absent from the controlled environment of a sleep laboratory. Moreover, not all parameters are recorded for all of the polysomnography studies, which restricts direct comparison and accurate estimates of SDB in patients with myelomeningocele.

\section{Conclusions}

A significant proportion of patients with myelomeningocele who had undergone polysomnography (81\%) were classified as having sleep apnea based on an initial sleep study. Furthermore, $31 \%$ had moderate or severe sleep apnea, predominately obstructive. Our results suggest that polysomnography in patients with myelomeningocele represents an opportunity to detect and classify sleep apnea, identify low-risk interventions, and prevent future implications of SDB.

\section{References}

1. Alsaadi MM, Iqbal SM, Elgamal EA, Gozal D: Sleep-disordered breathing in children with Chiari malformation type II and myelomeningocele. Pediatr Int 54:623-626, 2012

2. Bodenner KA, Jambhekar SK, Com G, Ward WL: Assessment and treatment of obstructive sleep-disordered breathing. Clin Pediatr (Phila) 53:544-548, 2014

3. Bowman RM, McLone DG, Grant JA, Tomita T, Ito JA: Spina bifida outcome: a 25-year prospective. Pediatr Neurosurg 34:114-120, 2001

4. Dauvilliers Y, Stal V, Abril B, Coubes P, Bobin S, Touchon J, et al: Chiari malformation and sleep related breathing disorders. J Neurol Neurosurg Psychiatry 78:1344-1348, 2007

5. Dennis M, Barnes MA: The cognitive phenotype of spina bifida meningomyelocele. Dev Disabil Res Rev 16:31-39, 2010

6. Gagnadoux F, Meslier N, Svab I, Menei P, Racineux JL: Sleep-disordered breathing in patients with Chiari malformation: improvement after surgery. Neurology 66:136-138, 2006

7. Gillespie MB, Flint PW, Smith PL, Eisele DW, Schwartz AR: Diagnosis and treatment of obstructive sleep apnea of the larynx. Arch Otolaryngol Head Neck Surg 121:335-339, 1995

8. Gozal D, Arens R, Omlin KJ, Jacobs RA, Keens TG: Peripheral chemoreceptor function in children with myelome- ningocele and Arnold-Chiari malformation type 2. Chest 108:425-431, 1995

9. Hesz N, Wolraich M: Vocal-cord paralysis and brainstem dysfunction in children with spina bifida. Dev Med Child Neurol 27:528-531, 1985

10. Hori T, Sugita Y, Koga E, Shirakawa S, Inoue K, Uchida S, et al: Proposed supplements and amendments to "A Manual of Standardized Terminology, Techniques and Scoring System for Sleep Stages of Human Subjects', the Rechtschaffen \& Kales (1968) standard. Psychiatry Clin Neurosci 55:305310, 2001

11. Kirk VG, Morielli A, Brouillette RT: Sleep-disordered breathing in patients with myelomeningocele: the missed diagnosis. Dev Med Child Neurol 41:40-43, 1999

12. Kirk VG, Morielli A, Gozal D, Marcus CL, Waters KA, D'Andrea LA, et al: Treatment of sleep-disordered breathing in children with myelomeningocele. Pediatr Pulmonol 30:445-452, 2000

13. Oren J, Kelly DH, Todres ID, Shannon DC: Respiratory complications in patients with myelodysplasia and Arnold-Chiari malformation. Am J Dis Child 140:221-224, 1986

14. Parker SE, Mai CT, Canfield MA, Rickard R, Wang Y, Meyer RE, et al: Updated National Birth Prevalence estimates for selected birth defects in the United States, 2004-2006. Birth Defects Res A Clin Mol Teratol 88:1008-1016, 2010

15. Putnam PE, Orenstein SR, Pang D, Pollack IF, Proujansky R, Kocoshis SA: Cricopharyngeal dysfunction associated with Chiari malformations. Pediatrics 89:871-876, 1992

16. Sandler AD: Children with spina bifida: key clinical issues. Pediatr Clin North Am 57:879-892, 2010

17. Sforza E, Roche F: Sleep apnea syndrome and cognition. Front Neurol 3:87, 2012

18. Sherman MS, Kaplan JM, Effgen S, Campbell D, Dold F: Pulmonary dysfunction and reduced exercise capacity in patients with myelomeningocele. J Pediatr 131:413-418, 1997

19. Sinha D, Guilleminault C: Sleep disordered breathing in children. Indian J Med Res 131:311-320, 2010

20. Swaminathan S, Paton JY, Ward SL, Jacobs RA, Sargent CW, Keens TG: Abnormal control of ventilation in adolescents with myelodysplasia. J Pediatr 115:898-903, 1989

21. Ward SL, Nickerson BG, van der Hal A, Rodriguez AM, Jacobs RA, Keens TG: Absent hypoxic and hypercapneic arousal responses in children with myelomeningocele and apnea. Pediatrics 78:44-50, 1986

22. Waters KA, Forbes P, Morielli A, Hum C, O'Gorman AM, Vernet O, et al: Sleep-disordered breathing in children with myelomeningocele. J Pediatr 132:672-681, 1998

\section{Author Contributions \\ Conception and design: Rocque, Patel, Hopson, Lozano. Acquisition of data: Patel, Hopson, Arynchyna, Bishop. Analysis and interpretation of data: Rocque, Patel. Drafting the article: Rocque, Patel, Hopson, Lozano. Critically revising the article: Rocque, Patel. Reviewed submitted version of manuscript: Rocque, Patel, Lozano, Blount. Approved the final version of the manuscript on behalf of all authors: Rocque. Statistical analysis: Rocque, Patel. Administrative/technical/material support: Patel, Hopson, Blount. Study supervision: Rocque, Patel, Blount.}

\section{Correspondence}

Brandon G. Rocque, 1600 Seventh Ave. S, Lowder 400, Birmingham, AL 35233. email: brandon.rocque@childrensal.org. 